\title{
DUAL TRANSITIVITY IN FINITE PROJECTIVE PLANES
}

\section{T. G. OSTROM}

Introduction. Let $\pi$ be a finite projective plane of order $n$,- i.e., with $n+1$ points per line. The author [2] has shown that if $\pi$ is doubly transitive and $n$ is an odd nonsquare, then $\pi$ is Desarguesian. M. Hall and D. Hughes, in a paper to appear soon, have removed the restriction that $n$ must be odd. In this paper, we show that if $\pi$ is dually transitive (see definition below), then it is doubly transitive.

\section{Dual transitivity.}

Definition. Let $p$ and $p_{1}$ be any two points, and let $L$ and $L_{1}$ be any two lines such that $p$ is not on $L$ and $p_{1}$ is not on $L_{1}$. If (for all choices of $\left.p, p_{1}, L, L_{1}\right)$ there is a collineation of $\pi$ which carries $p$ into $p_{1}$ and $L$ into $L_{1}$, then $\pi$ will be said to be dually transitive.

Theorem 1. Let $p$ and $p_{2}$ be any two points, and let $L$ and $L_{2}$ be any two lines such that $p$ is not on $L$ and $p_{2}$ is not on $L_{2}$. If (for all choices of $\left.p, p_{2}, L, L_{2}\right)$ there is a correlation of $\pi$ which carries $p$ into $L$ and $p_{2}$ $i^{\text {nto }} L_{2}$ then $\pi$ is dually transitive.

Proof. Consider the collineations which arise by taking the products of two correlations.

Lemma 1. Suppose that the projective plane $\pi$ of order $n$ admits a group $\Sigma$ of collineations which (1) leaves a certain line $L$ fixed and (2) is transitive on points not belonging to $L$, then if $L_{1}$ and $L_{2}$ are two lines $\neq L$ whose point of intersection lies on $L$, there is a collineation of $\Sigma$ which carries $L_{1}$ into $L_{2}$.

Proof. Let us refer to points not on $L$ as ordinary points. Let $\Subset$ denote a transitive class of lines under $\Sigma$-i.e., each line of $\mathbb{5}$ can be carried into each other line of $\mathbb{S}$ by a collineation of $\Sigma$ and no line not in $\mathbb{S}$ is the image of any line in $\mathbb{S}$. Now let $p$ be an ordinary point which is on exactly $k$ lines of $\mathbb{E}$. The collineation which carries $p$ into some other ordinary point $p_{1}$ carries the above mentioned $k$ lines into $k$ lines through $p_{1}$. Hence every ordinary point lies on exactly $k$ lines of $\mathbb{E}$. Let $m$ denote the total number of lines in $\mathfrak{5}$. Since each line of $\mathfrak{E}$ contains $n$ ordinary points, and there are $n^{2}$ ordinary points in all, it follows that

$$
m n=k n^{2}, \quad \text { or } \quad m=k n .
$$

Presented to the Society June 15, 1957; received by the editors January 17, 1957 and, in revised form, June 21, 1957. 
Now let $L_{1}$ be some line of $\mathbb{E}$, intersecting $L$ in the point $q$. Each of the $n$ ordinary points of $L_{1}$ lies on $k-1$ lines of $\mathbb{S}$ other than $L_{1}$ itself. This accounts for $n(k-1)$ lines of $(5$. The remaining $n$ lines must all go through $q$. Thus all of the ordinary lines which pass through any point $q$ on $L$ must belong to the same transitive class and the lemma is proved.

Theorem 2. If $\pi$ is dually transitive, it is doubly transitive.

Proof. It follows from Lemma 1 that, given any line $L$ and any point $p$ on $L$, the group of collineations which leaves $p$ and $L$ fixed is transitive on the other lines through $p$. Now if $p$ is on the lines $L$ and $L_{1}$, by considering the product of the two groups which fix $p$ and $L$ on one hand, and $p$ and $L_{1}$ on the other hand, we see that the group which fixes $p$ is transitive on lines through $p$. The dual transitivity implies that the group $\Sigma$ which leaves $p$ fixed is transitive on lines not through $p$. Thus, there are exactly two transitive classes of lines under the group $\Sigma$. Parker [3] has shown that the number of point transitivity classes under a group of collineations is equal to the number of line classes. Since $p$ is in a class by itself, all other points lie in a single transitive class under $\Sigma$. This in turn implies that any two points can be carried into any two points. (It should be noted here that D. R. Hughes has communicated a similar proof to the author.)

\section{REFERENCES}

1. A. M. Gleason, Finite Fano planes, Amer. J. Math. vol. 78 (1956) pp. 797-807.

2. T. G. Ostrom, Double transitivity in finite projective planes, Canadian J. Math. vol. 8 (1956) pp. 563-567.

3. E. T. Parker, On collineations of symmetric designs, Proc. Amer. Math. Soc. vol. 8 (1957) pp. 250-351.

Montana State University 